\title{
Isolated ATP synthase deficiency
}

INSERM

\section{Source}

INSERM. (1999). Orphanet: an online rare disease and orphan drug data base. Isolated ATP synthase deficiency. ORPHA:254913

Isolated ATP synthase deficiency is a rare, genetic, mitochondrial oxidative phosphorylation disorder that may present with a wide range of symptoms (including muscular hypotonia, hypertrophic cardiomyopathy, psychomotor delay, encephalopathy, peripheral neuropathy, lactic acidosis, 3-methylg lutaconic aciduria) and clinical syndromes (including NARP and MILS). 